\title{
GREEN'S SECOND IDENTITY FOR GENERALIZED LAPLACIANS
}

WALTER RUDIN

Let $J(P, r)$ denote the closed circular disc bounded by the circle $C(P, r)$ with center at $P$, and radius $r$, in the plane. We define the generalized Laplacian of the function $F$ at $P$ by

$$
\Lambda F(P)=\lim _{r \rightarrow 0} 4[m(F ; P, r)-F(P)] / r^{2},
$$

where $m(F ; P, r)$ is the mean value of $F$ on $C(P, r)$. The upper and lower Laplacians $\Lambda^{*} F(P)$ and $\Lambda_{*} F(P)$ are defined likewise, with lim sup and $\lim$ inf in place of $\lim [3] .{ }^{1}$ If $f \in L$ in a bounded domain $R$, we define $[3$, p. 281]

$$
\Omega_{R} f(P)=-\frac{1}{2 \pi} \iint_{R} f(Q) g(P, Q) d Q \quad(P \text { in } R),
$$

where $g(P, Q)$ is Green's function for $R$. In [3] we established the existence of $\Lambda F(P)$ for almost all $P$ of a domain $D$, the integrability of $\Lambda F(P)$ over any compact subset of $D$, and the formula

$$
F(P)=\Omega_{R} \Lambda F(P)+H(P),
$$

valid for almost all $P$ of any bounded domain $R$ such that $\bar{R} \subset D$, where $H$ is harmonic in $R$ and assumes the values of $F$ on the boundary of $R$, under the following hypotheses:

(A) $F$ is continuous in $D$;

(B) $\Lambda^{*} F(P)>-\infty, \Lambda_{*} F(P)<+\infty$, except possibly on a closed set of capacity zero;

(C) there exists a function $y$, defined in $D$, such that $y \in L$ on every compact subset of $D$, and such that $y(P) \leqq \Lambda^{*} F(P)$ for $P$ in $D$.

In [4], (B) was slightly weakened. In the present paper the above result is used to obtain the following theorem.

THEOREM. If the functions $U$ and $V$ satisfy (A), (B), (C) in a domain $D$, and if $U(P)=0$ outside a compact subset $K$ of $D$, then

$$
\iint_{D} U(P) \Lambda V(P) d P=\iint_{D} V(P) \Lambda U(P) d P .
$$
1950

Presented to the Society, February 24, 1951; received by the editors December 11,

${ }^{1}$ Numbers in brackets refer to the references at the end of the paper. 
If $U$ and $V$ have continuous second derivatives, then (2) is clearly an immediate consequence of Green's second identity [1, p. 215].

Let $R$ be a bounded domain such that $K \subset R \subset \bar{R} \subset D$. Since $U$, and therefore $\Lambda U$, vanish outside $K$, it suffices to prove that (2) holds with $R$ in place of $D$.

Putting $u(P)=\Lambda U(P), v(P)=\Lambda V(P)$, wherever the Laplacians exist, we have, by (1), writing $\Omega$ for $\Omega_{R}$,

$$
U(P)=\Omega u(P), \quad V(P)=\Omega v(P)+H(P) \quad \text { (p.p. in } R) .
$$

By Fubini's theorem, and (3),

$$
\begin{aligned}
\iint_{R} v(P) \Omega u(P) d P & =\iint_{R} u(P) \Omega v(P) d P \\
& =\iint_{R} u(P) V(P) d P-\iint_{R} u(P) H(P) d P .
\end{aligned}
$$

Hence it is enough to prove that

$$
\iint_{R} u(P) H(P) d P=0
$$

for every function $H$ harmonic in $R$. Choose a domain $G$ such that $K \subset G \subset \bar{G} \subset R$. Choose $r>0$ such that $J(P, 3 r) \subset R$ if $P \in G$. Define $H(P)=0$ outside $R$. Put $H_{1}(P)=A_{r} H(P)$ (that is, the mean of $H$ on $J(P, r)), H_{2}(P)=A_{r} H_{1}(P)$, and $H_{3}(P)=A_{r} H_{2}(P)$, for all $P$. Then $H_{3}(P)=H(P)$ in $G, H_{3}$ has continuous second derivatives everywhere [2, p. 343], and $H_{3}(P)=0$ outside some bounded domain $T$ containing $\bar{G}$. Hence we have, for all $P$ in $T$,

$$
H_{3}(P)=\Omega_{T} h_{3}(P),
$$

where $h_{3}(P)=\Lambda H_{3}(P)$. Noting that $u(P)=U(P)=0$ wherever $H(P)$ $\neq H_{3}(P)$, we obtain

$$
\begin{aligned}
\iint_{R} u(P) H(P) d P & =\iint_{T} u(P) \Omega_{T} h_{3}(P) d P \\
& =\iint_{T} h_{3}(P) \Omega_{T} u(P) d P=\iint_{T} U(P) \Lambda H(P) d P=0,
\end{aligned}
$$

since $\Lambda H(P)=0$ in $K$, and $U(P)=0$ in $T-K$. This proves (4), and hence the theorem. 
The extension to more than two dimensions is evident.

\section{REFERENCES}

1. O. D. Kellogg, Foundations of potential theory, New York, 1929.

2. F. Riesz, Sur les fonctions subharmoniques et leur rapport d la theorie du potentiel, Acta Math. vol. 54 (1930) pp. 321-360.

3. W. Rudin, Integral representation of continuous functions, Trans. Amer. Math. Soc. vol. 68 (1950) pp. 278-286.

4. - $A$ theorem on subharmonic functions, Proceedings of the American Mathematical Society vol. 2 (1951) pp. 209-212.

Massachusetts Institute of Technology 